\title{
Photopatternable, Branched Polymer Hydrogels Based on Linear Macromonomers for 3D Cell Culture Applications
}

\author{
Ciqing Tong, Joeri A. J. Wondergem, Doris Heinrich, and Roxanne E. Kieltyka*
}

Cite This: ACS Macro Lett. 2020, 9, 882-888

Read Online

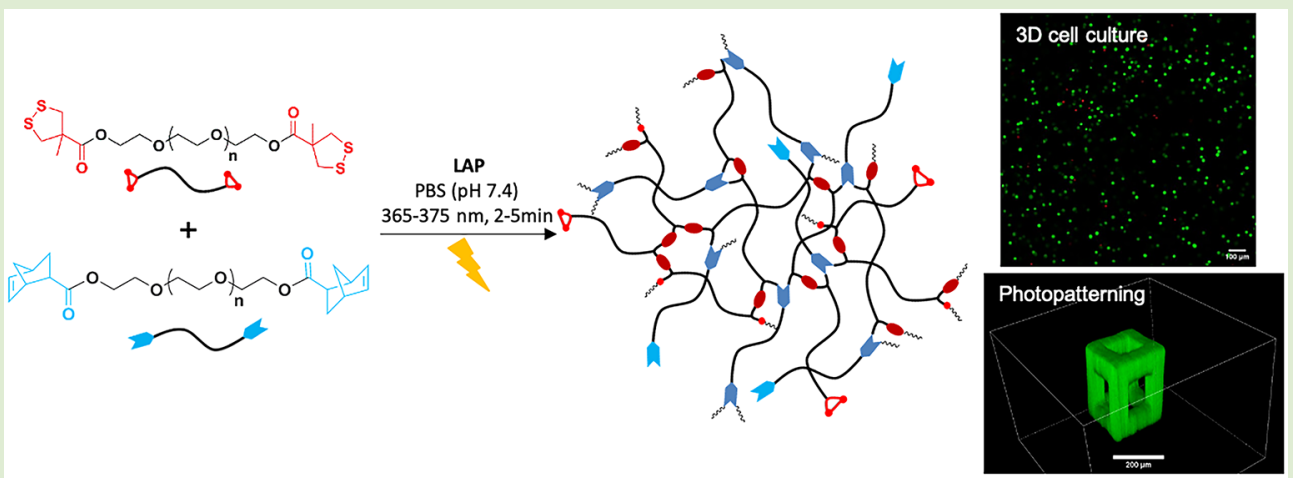

ABSTRACT: Photochemical ligation strategies in hydrogel materials are crucial to model spatiotemporal phenomena that occur in the natural extracellular matrix. We here describe the use of cyclic 1,2-dithiolanes to cross-link with norbornene on linear poly(ethylene glycol) polymers through UV irradiation in a rapid and byproduct-free manner, resulting in branched macromolecular architectures and hydrogel materials from low-viscosity precursor solutions. Oscillatory rheology and NMR data indicate the one-pot formation of thioether and disulfide cross-links. Spatial and temporal control of the hydrogel mechanical properties and functionality was demonstrated by oscillatory rheology and confocal microscopy. A cytocompatible response of NIH 3T3 fibroblasts was observed within these materials, providing a foothold for further exploration of this photoactive cross-linking moiety in the biomedical field.

ynthetic hydrogels have gained attention as scaffolds to $\checkmark$ mimic the 3D microenvironment of cells in vitro thanks to their water-rich character in conjunction with their tunable chemical and physical properties. ${ }^{1}$ To this end, highly hydrophilic poly(ethylene glycol) (PEG)-based polymers combined with various cross-linking methods (e.g., photopolymerization, chemoselective ligation, enzyme-mediated cross-linking) have been exploited to prepare hydrogels for $3 \mathrm{D}$ cell culture for a diverse array of cell types and aims. ${ }^{2-4}$ Most reported examples are based on branched (multiarmed or star) precursors; far fewer involve cross-linking of endfunctionalized linear chains that are synthetically accessible, but typically require additional components to form gel-phase materials. $^{5-11}$ Therefore, bio-orthogonal and atom-efficient cross-linking chemistries that provide handles for external control of the reaction rate remain attractive to form and modulate hydrogel properties in the biomedical area. ${ }^{12-14}$

Thiol-based chemistries have seen extensive use in the polymer field for the vulcanization of rubbers and more recently, for bioconjugation. ${ }^{15-18}$ As an alternative to metalbased bioconjugations, thiol-X reactions have grown in use because of their efficiency under mild reaction conditions, following either a nucleophilic addition-elimination or a radical mechanism. Both mechanisms have been applied in the hydrogel field, ${ }^{19,20}$ the latter often being initiated by photoactivation to enable chemical cross-linking or polymerization. $^{21-23}$

A drawback to the use of thiols is that their reactivity often results in disulfide formation during storage, which can be problematic for their subsequent application. ${ }^{24}$ Consequently, protected and latent thiols are being increasingly used in polymer materials to gain control over their properties. ${ }^{24}$ Thiol protection strategies based on linear disulfides are atominefficient, releasing small molecules that can leach out of the material or require additional purification steps, which could be problematic for use in the biomedical field. ${ }^{24}$ In contrast, latent thiols that reveal the reactive moiety on demand are highly attractive because of their atom-efficient nature and lack of byproducts. In the polymer chemistry field, cycles based on carbon-sulfur bonds (e.g., thiolactones, Traut's reagent) have

Received: March 2, 2020

Accepted: May 6, 2020

Published: June 1, 2020 


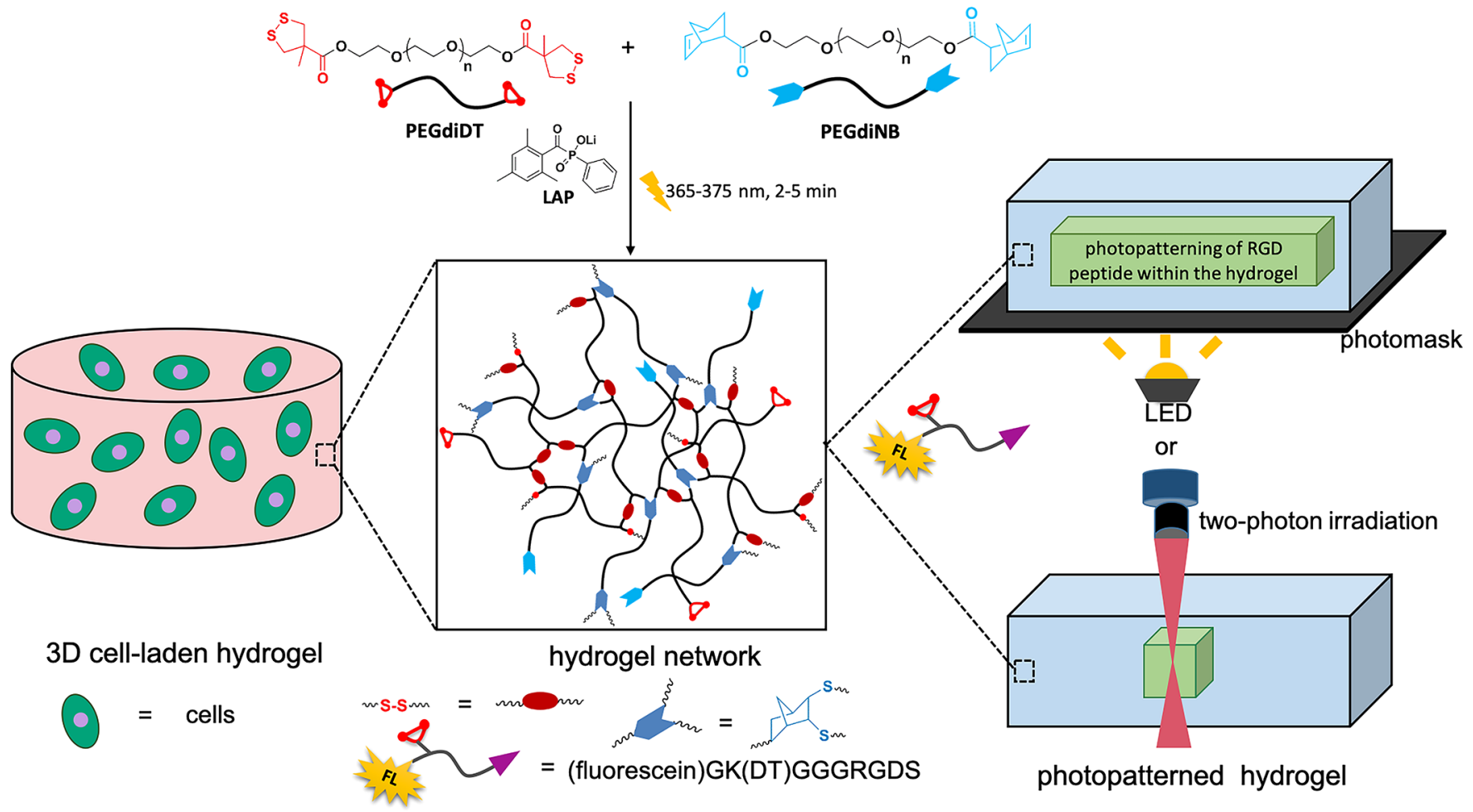

Figure 1. Photoreaction of DT and NB polymers in the presence of a cell suspension results in hydrogels with cells encapsulated in 3D. They can be further patterned with a fluorescently-labelled RGD peptide using a photomask during UV irradiation or two-photon laser lithography.
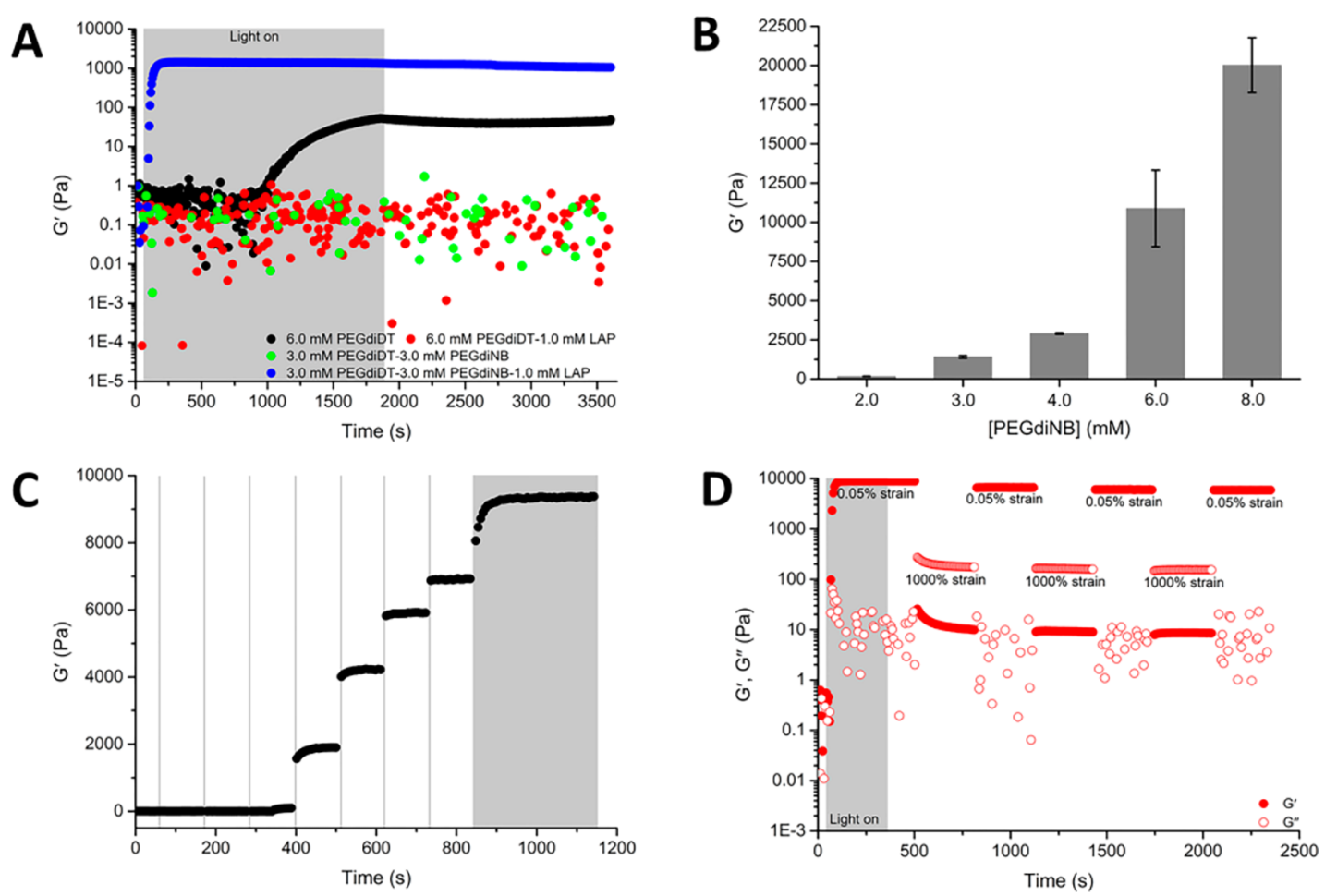

Figure 2. (A) Averaged $(\mathrm{N}=3)$ time sweep of PEGdiDT hydrogels and PEGdiDT-PEGdiNB hydrogels without and with LAP (1.0 mM) using 30 min UV irradiation during the measurement $(\gamma=0.05 \%, f=1.0 \mathrm{~Hz})$ at room temperature. (B) The plateau storage moduli $\left(G^{\prime}\right)$ of hydrogels PEGdiDT-PEGdiNB with LAP $(1.0 \mathrm{mM})$ were tuned by adjusting the total polymer concentration and keeping the ratio of $[\mathrm{NB}] /[\mathrm{DT}](1: 1)$ constant (under $5 \mathrm{~min}$ UV irradiation). (C) Step UV-light irradiation of a hydrogel containing $6.0 \mathrm{mM}$ PEGdiDT-6.0 mM PEGdiNB-1.0 mM LAP. (D) Averaged $(N=3)$ step-strain measurement of hydrogel $(6.0 \mathrm{mM}$ PEGdiDT-6.0 mM PEGdiNB-1.0 mM LAP) after $5 \mathrm{~min}$ UV irradiation. For all data, UV-light irradiation condition: $10 \mathrm{~mW} / \mathrm{cm}^{2}$, wavelength: $320-500 \mathrm{~nm}$, primary peak: $365 \mathrm{~nm}$. The shaded part of the data indicates when light was applied. Error bars were calculated according the average of repeat measurements $(N \geq 3)$. 
been extensively explored as latent thiols, ${ }^{24}$ whereas far fewer examples involving cyclic disulfides (e.g., 1,2-dithiolanes, 1,2dithianes $)^{25-30}$ have been reported. The latter can be especially beneficial in gel phase materials, thanks to their capacity to act as bifurcated cross-linkers or cargo attachment points providing two reactive thiol moieties on ring opening. ${ }^{31-34}$ Thus far, most approaches using cyclic disulfides use conditions that favor nucleophilic addition-elimination, resulting in materials that require additional small molecule reagents for their stabilization and can prove challenging for their application in biomaterials. ${ }^{31,32}$

In this study, we exploit the instability of 1,2-dithiolanes (DT) using methyl asparagusic acid (Figures S1 and S2) with UV light at $365-375 \mathrm{~nm}$ to prepare hydrogel materials with spatiotemporal control over their properties in an atomefficient manner. We specifically examine their introduction on linear PEG polymers to understand their capacity to prepare light-responsive hydrogel materials for 3D cell culture (Figure 1) in an easy and economic way.

Linear PEG polymers (PEGdiDT, PEGdiNB) were synthesized by a one-step reaction using carbodiimide coupling chemistry resulting in a high degree of end-functionalization (ca. 100\%; Figures S3 and S5). To examine the potential for UV-mediated cross-linking of the DT moiety, UV irradiation at either 365 or $375 \mathrm{~nm}$ (depending on the light source) was applied, using the absorption of DT that has a maximum at 330 $\mathrm{nm}$. Importantly, irradiation of the linear macromonomer PEGdiDT on its own or in combination with linear PEGdiNB and a photoinitiator, lithium phenyl-2,4,6-trimethylbenzoylphosphinate (LAP), resulted in hydrogel materials starting from low viscosity precursor solutions (vide infra).

Oscillatory rheology was used to evaluate the gelation rate and mechanical properties of the photo-cross-linked DT-NB PEG hydrogels. Because of the known capacity of the DT moiety to form disulfide cross-links or undergo polymerization on its own in the presence of nucleophiles, heat or light, ${ }^{33,35,36}$ we first evaluated the capacity of the PEGdiDT polymer to cross-link with itself prior to the addition of PEGdiNB. PEGdiDT $(6.0 \mathrm{mM})$ required more than $15 \mathrm{~min}$ UV irradiation to yield a viscous solution, and $30 \mathrm{~min}$ for a weak hydrogel (storage modulus $G^{\prime}=42 \pm 6 \mathrm{~Pa}$ ). Moreover, addition of LAP $(1.0 \mathrm{mM})$ to PEGdiDT did not yield a hydrogel despite its reaction with DT (vide infra). Thus, the inefficient gelation of PEGdiDT on its own could be challenging for applications involving $3 \mathrm{D}$ cell culture.

In contrast, replacing half of the macromonomer $(3.0 \mathrm{mM})$ with PEGdiNB and using LAP $(1.0 \mathrm{mM})$ resulted in a drastic reduction of the gelation time (less than $2 \mathrm{~min}$ ) and a substantial increase of the storage modulus $\left(G^{\prime}=1265 \pm 62\right.$ Pa; Figure 2A). Without LAP, no hydrogel was formed after 30 min UV irradiation (Figure 2A). Also, no hydrogel was formed when cross-linking was attempted with PEGdiSH and PEGdiNB as a control, unless a multi-arm cross-linker (PEG4SH) was used, pointing out the importance of DT for network formation from linear macromonomers (Figure S21). Moreover, increasing the total polymer concentration (from 4.0 to $16.0 \mathrm{mM}$ ) while maintaining an equimolar DT/NB ratio and constant LAP concentration $(1.0 \mathrm{mM})$ resulted in highly efficient hydrogel formation, with $\mathrm{G}^{\prime}$ at the plateau rising from $167 \pm 11 \mathrm{~Pa}$ to $20018 \pm 1781 \mathrm{~Pa}$ (Figure 2B and S22). Hence, DT can efficiently form hydrogels with $\mathrm{NB}$ on linear macromonomers upon light irradiation in the presence of a photoinitiator at a rate that is attractive for use in $3 \mathrm{D}$ cell culture.

To better understand the scope of the photoactivated onepot DT-NB reaction for the preparation of hydrogels, we measured their rheological properties while tuning the LAP concentration, light intensity, step light irradiation time, and $[\mathrm{NB}] /[\mathrm{DT}]$ ratio (Table S5). Using the lowest LAP concentration $(0.1 \mathrm{mM})$ and $5 \mathrm{~min}$ UV irradiation, no gelation was observed. Increasing LAP $(1.0 \mathrm{mM})$, provided a higher rate of gel formation and an increase in $G^{\prime}$, while higher LAP concentrations $(4.0 \mathrm{mM})$ did not yield a greater increase in $G^{\prime}$ (Figure S23). A higher intensity of the light source ( 30.0 $\left.\mathrm{mW} / \mathrm{cm}^{2}\right)$ increased the rate of gel formation in comparison to lower values $\left(\sim 5.0 \mathrm{~mW} / \mathrm{cm}^{2}, \sim 10.0 \mathrm{~mW} / \mathrm{cm}^{2}\right)$, but in all cases, a comparable $G^{\prime}$ was eventually reached (Figure S24). Moreover, the $G^{\prime}$ of the hydrogels can be modulated in a stepwise fashion using UV irradiation in intervals, demonstrating the potential of this chemistry for applications where spatial or temporal control is desired (Figure 2C). Increasing the concentration of DT relative to NB ([NB]/[DT] from $2: 1$ to $1: 2$ ) resulted in hydrogel formation and an increase in $G^{\prime}$, while further increasing DT $([\mathrm{NB}] /[\mathrm{DT}]=1: 3)$ resulted in a slower gelation rate and decreased $G^{\prime}$ (Figure S25).

To probe the contribution of the reversible covalent crosslinks between the DT moieties to the properties of the DT-NB hydrogels, we evaluated their potential for self-recovery through a step-strain experiment. All hydrogels showed a recovery of $50-70 \%$ of the original $G^{\prime}$ after the application of high strain, with the recovery increasing with DT concentration due to an increase in the number of dynamic disulfide cross-links in the network (Figures 2D and S26).

Scanning electron microscopy was performed to gain insight into the hydrogel microstructure. A macroporous structure of the PEGdiDT-PEGdiNB hydrogel was observed, consistent with other covalent polymer hydrogels (Figure S27). Additionally, to shed light on the degradation kinetics of the PEGdiDT and PEGdiDT-PEGdiNB hydrogels, their swelling ratio was examined at various $[\mathrm{NB}] /[\mathrm{DT}]$ ratios at different time points (Figure S28). The swelling ratio of the DT-NB hydrogel for $[\mathrm{NB}] /[\mathrm{DT}]$ ratios of comparable $G^{\prime}$ remained largely unchanged after 9 days in PBS or cell culture media (DMEM) at $37^{\circ} \mathrm{C}$. Conversely, PEGdiDT hydrogels degraded within $24 \mathrm{~h}$, highlighting the importance of using NB in the polymer networks to enable their use for cell culture applications.

Collectively, these results suggests that the irradiation of DT with UV light forms both thioether and disulfide bonds due to the reaction of the NB and DT units, respectively, enabling the formation of hydrogel materials from linear polymer precursors that are inaccessible using linear disulfides or monothiols.

To further understand the DT-NB reaction on the PEG macromonomers in the presence of LAP, ${ }^{1} \mathrm{H}$ NMR spectroscopy was performed in $\mathrm{D}_{2} \mathrm{O}$. Monofunctional linear polymers (PEGmDT and PEGmNB, Figures S4 and S6) were synthesized starting from $O$-methyl-undecaethylene glycol in an effort to avoid hydrogel formation during the reaction and to simplify NMR analysis. UV light activation of PEGmDT or PEGmNB on their own, with and without LAP was first examined (Figures S7-S15 and Tables S1 and S2). For all reactions, the $-\mathrm{CH}_{3}$ peak $(\sim 3.35 \mathrm{ppm})$ of $O$-methylundecaethylene glycol was selected as a reference, and changes in the $-\mathrm{CH}_{2}(\sim 3.01-3.04 \mathrm{ppm})$ and $-\mathrm{CH}_{3}(\sim 1.49 \mathrm{ppm})$ peaks of the DT and the -ene proton signals ( $\sim 5.92-6.24$ 


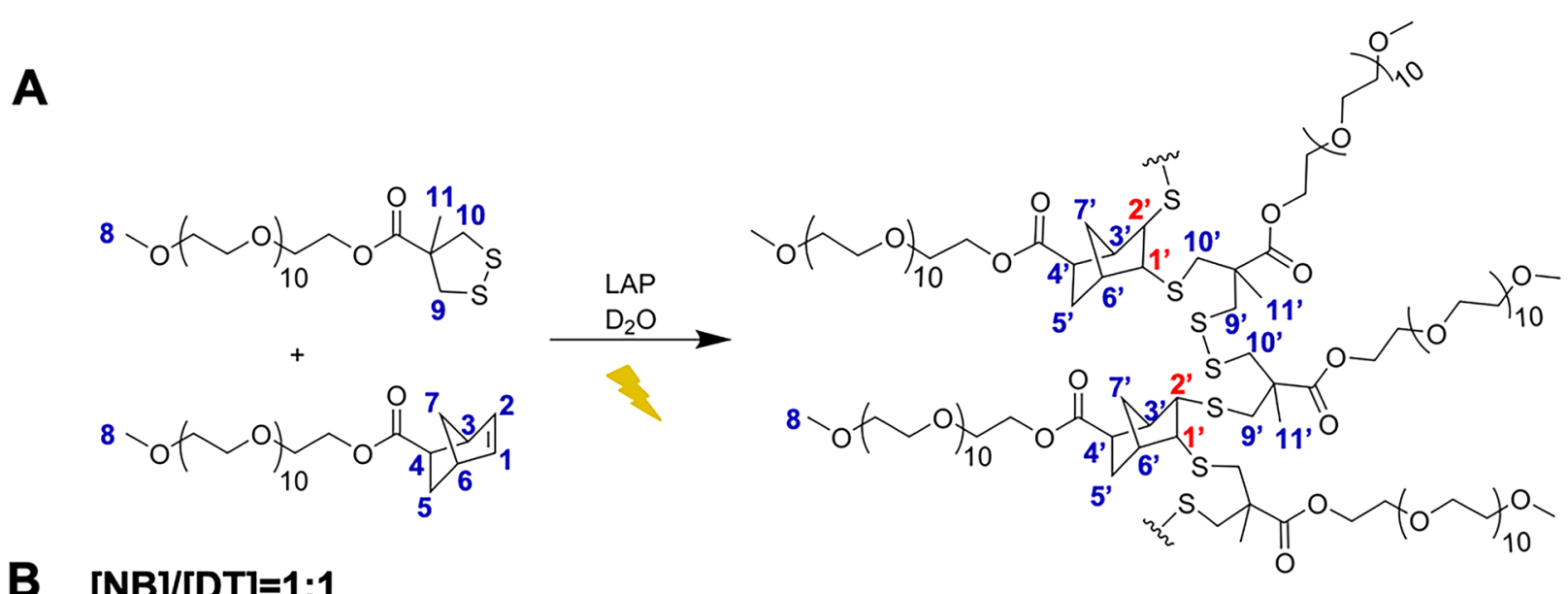

\section{B $[\mathrm{NB}] /[\mathrm{DT}]=1: 1$}

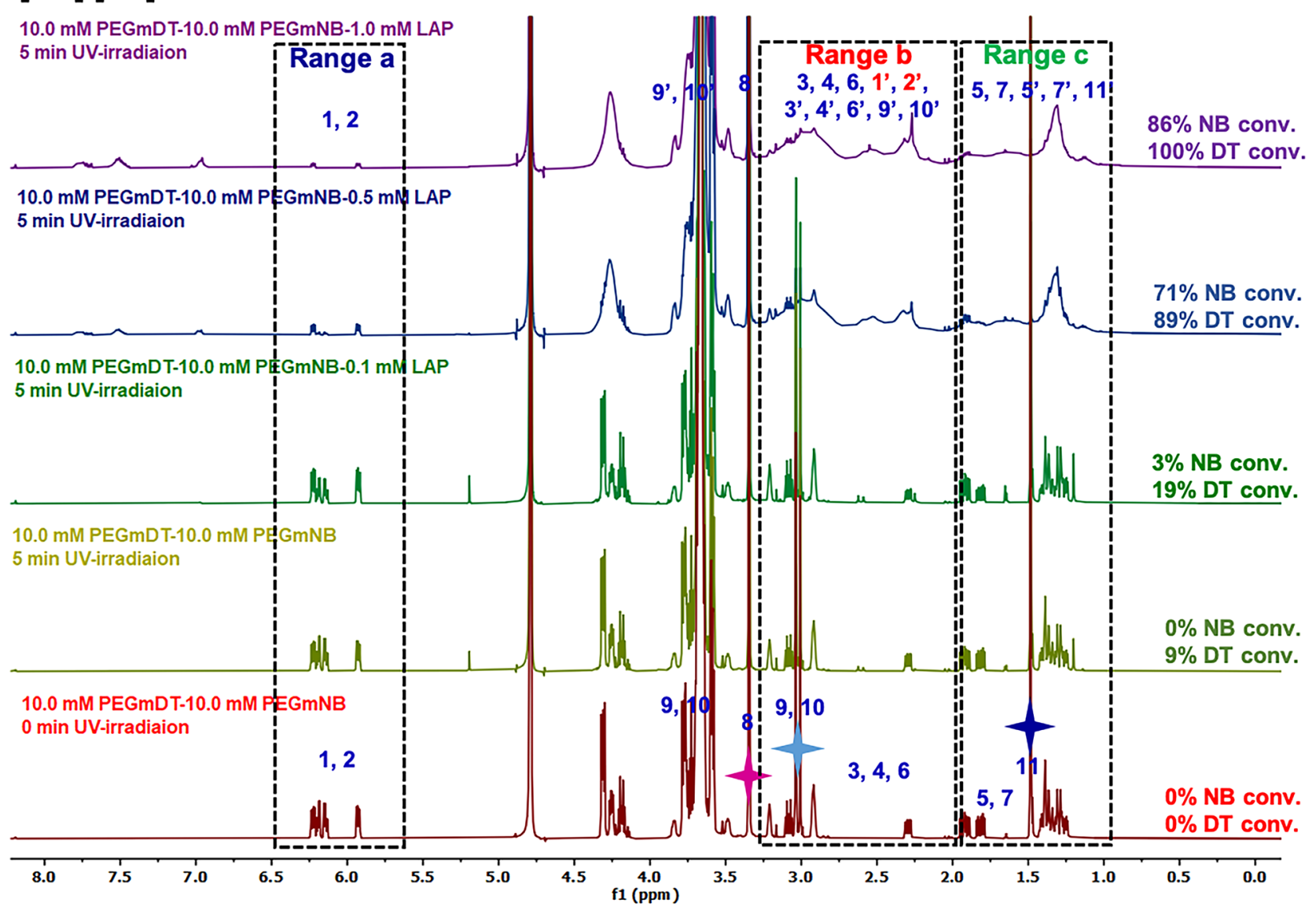

Figure 3. (A) Proposed reaction product of PEGmDT and PEGmNB, including double substitution of norbornene and disulfide cross-links, based on ${ }^{1} \mathrm{H}$ NMR and oscillatory rheology. (B) ${ }^{1} \mathrm{H}$ NMR $(400 \mathrm{MHz})$ spectra in $\mathrm{D}_{2} \mathrm{O}$ of the PEGmDT-PEGmNB reaction $([\mathrm{NB}] /[\mathrm{DT}]=1: 1)$ with different concentrations of LAP $(0,0.1,0.5$, and $1.0 \mathrm{mM})$ without and with $5 \mathrm{~min}$ UV irradiation using a benchtop LED $\left(\sim 10 \mathrm{~mW} / \mathrm{cm}^{2}, 375 \mathrm{~nm}\right)$.

ppm, range a) of NB were used to calculate the conversion of $\mathrm{DT}$ and $\mathrm{NB}$, respectively, during the reaction. Whereas no change in the ${ }^{1} \mathrm{H}$ NMR spectrum for PEGmNB was observed, even after 30 min UV light, irradiation of PEGmDT for 5-30 min resulted in the formation of multiple new peaks $(\sim 1.99-$ $3.30 \mathrm{ppm}$, range $\mathrm{b}$; and $\sim 1.00-1.98 \mathrm{ppm}$, range $\mathrm{c}$ ) upfield of the $-\mathrm{CH}_{2}$ and $-\mathrm{CH}_{3}$ resonances of DT, suggestive of its ringopening polymerization. Addition of an increasing concentration of LAP to either PEGmNB or PEGmDT accelerated the consumption of NB (e.g., $3 \%$ conv. for 1.0 mM LAP and $\sim 47 \%$ conv. for $10.0 \mathrm{mM} \mathrm{LAP})$ and DT ( 17\% conv. for 1.0 mM LAP and $\sim 72 \%$ conv. for $10.0 \mathrm{mM} \mathrm{LAP}$ ) with short UV irradiation times $(5 \mathrm{~min})$, pointing out the reaction of the initiator with both of the individual components. This result is consistent with an earlier report by Anseth et al. that showed that high concentrations of LAP can consume the disulfides of hydrogel precursors. ${ }^{37}$

Using an equimolar solution of PEGmNB and PEGmDT with $5 \mathrm{~min}$ UV irradiation resulted in increased conversion with higher LAP concentrations (0.1 mM LAP: 3-16\% conv. of NB and $\sim 12-19 \%$ conv. of DT; 1.0 mM LAP: $\sim 80-$ $86 \%$ conv. of $\mathrm{NB}$ and $\sim 100 \%$ conv. of DT). New peaks were found to appear between 2.0 and $3.0 \mathrm{ppm}$, as previously reported for thioether $\alpha$-protons, ${ }^{23}$ further confirming the 
A
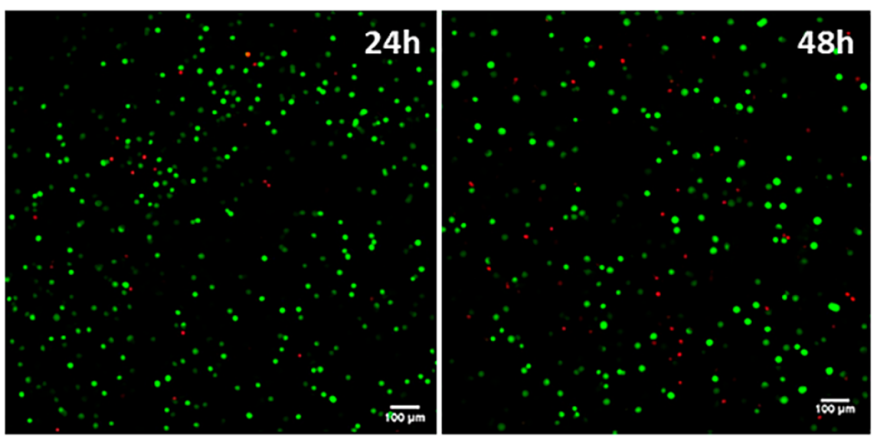

C

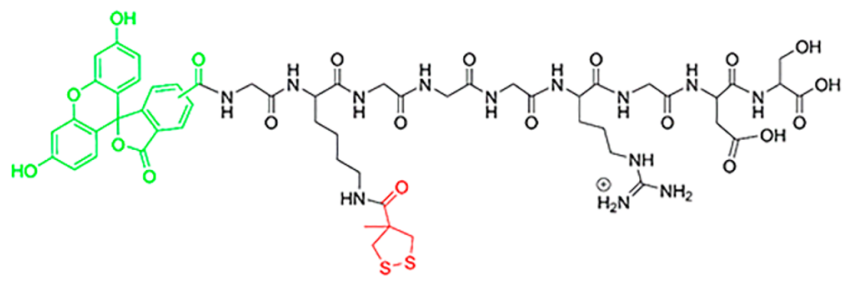

(Fluorescein)GK(DT)GGGRGDS
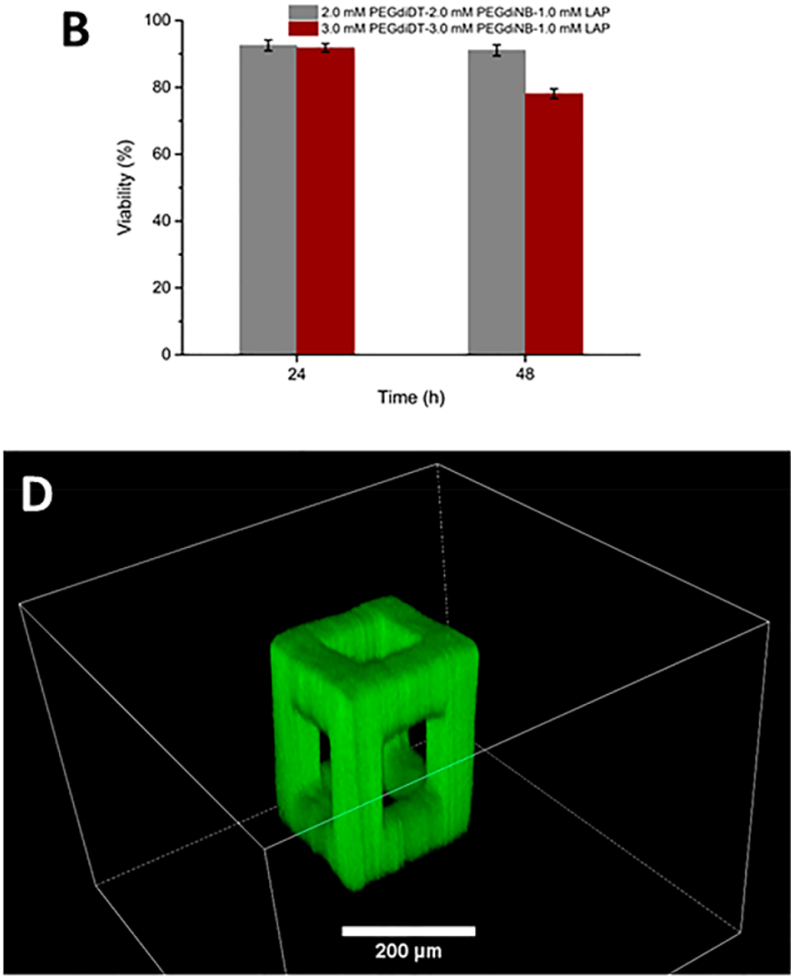

Figure 4. (A) Representative confocal microscopy images of NIH 3T3 cells after 24 and $48 \mathrm{~h}$ encapsulated in $3 \mathrm{D}$ of the DT-NB hydrogel (3.0 mM PEGdiDT-3.0 mM PEGdiNB-1.0 mM LAP) with 2 min UV irradiation using a benchtop LED $\left(\sim 10 \mathrm{~mW} / \mathrm{cm}^{2}, 375 \mathrm{~nm}\right)$. Scale bar: $100 \mu \mathrm{m}$. Green: viable cells, red: dead cells. (B) Averaged $(N=3)$ cell viabilities of NIH 3 T3 cells at 24 and 48 h. Error bars represent the standard deviation of independent samples. (C) Chemical structure of the fluorescein-labeled cell-adhesive RGD peptide: (fluorescein)GK(DT)GGGRGDS. (D) Confocal microscopy image of two-photon cross-linked and a bound cell-adhesive peptide through DT-NB (3.0 mM PEGdiDT-3.0 mM PEGdiNB-1.0 mM LAP) via direct-laser writing. Scale bar: $200 \mu \mathrm{m}$.

bond formation between the DT and NB units. Substantial broadening of all peaks in the NMR spectrum suggest the polymerization of both components (Figures 3 and S16 and Table S3).

In a second step, we evaluated the photoinitiated DT-NB reaction with LAP $(1.0 \mathrm{mM})$ at different $[\mathrm{NB}] /[\mathrm{DT}]$ ratios (2:1 to $1: 2)$ to gain insight into cross-links that drive the formation of the hydrogel material (Figures S17 and S18 and Table S3). Complete NB conversion (from $43 \%$ to $100 \%$ ) after 5 min UV irradiation was obtained when increasing the DT concentration. This result shows that an excess of DT is necessary to fully cross-link NB and suggests that disulfide bond formation, and inactivation of DT by LAP occur in parallel in the hydrogel.

As a control experiment, we compared the integration of regions a-c from the DT-NB reaction against a reaction of PEGmNB with DL-dithiothreitol (DTT), a dithiol reagent that forms hydrogen radicals with UV light (5.0 mM DTT-10.0 mM PEGmNB-1.0 mM LAP, Figure S19). After $5 \mathrm{~min}$ irradiation, a decrease in the integrated area of range a, followed by a symmetrical increase in the integrated areas of ranges $b$ and $c$ were observed (Table S4). This result is consistent with a thiol-ene reaction, where the loss of the NBene signals in range a (signals 1 and 2) and the simultaneous formation of a single thioether linkage in range $b$ (signal 1 ') and $\mathrm{a}-\mathrm{CH}_{2}-$ group in range $\mathrm{c}$ (signal $2^{\prime}$ ) are expected upon UV light irradiation. ${ }^{23}$ In comparison, in the DT-NB reaction, the reduction of the integrated area of range a (disappearance of the -ene in NB) and the concomitant increase of range $b$ while range $c$ remains unchanged, suggest the formation of two thioether bonds on NB (Table S3). This result is made possible by the lack of abstractable $-\mathrm{SH}$ protons and the formation of two thiyl radicals with UV light on the DT moiety.

We then examined the potential for application of the DT$\mathrm{NB}$ reaction for $3 \mathrm{D}$ cell culture applications by encapsulating $\mathrm{NIH}$ 3T3 fibroblasts in the hydrogel materials. Initial gel inversion experiments showed that PEGdiDT-PEGdiNB hydrogels were formed in cell culture media (Figure S20), opening the door for further experiments involving cells. When hydrogels consisting of $2.0 \mathrm{mM}$ PEGdiDT-2.0 $\mathrm{mM}$ PEGdiNB/1.0 mM LAP that were irradiated with UV light for 2 minutes, largely viable cell populations (>91\%) were observed in a LIVE/DEAD assay after 24 and $48 \mathrm{~h}$ (Figure S29). However, increasing the overall polymer concentration $(6.0 \mathrm{mM})$ decreased cell viability to $\sim 80 \%$ after $48 \mathrm{~h}$ culture (Figure 4A,B). Generally, a cytocompatible response of $\mathrm{NIH}$ $3 \mathrm{~T} 3$ cells in the presence of the DT-NB cross-linking reaction was found.

Next, we further examined the potential of the DT-NB reaction for spatial and temporal photopatterning of hydrogel materials for cell culture applications. A fluorescein dye- and DT-labeled cell-adhesive RGD peptide, (fluorescein)GK(DT)GGGRGDS (Figure 4C), was synthesized to visualize and study the possibility of UV-light-activated coupling between the hydrogel and functional peptides, such as RGD. After initial UV exposure to form the hydrogel, secondary illumination through a photomask produced RGD-hydrogel patterns with cell-size features throughout the volume of the gel (Figure S30). Moreover, the low near-infrared absorbance 
of the hydrogel enables full spatial control over peptide-gel photo coupling by two-photon laser lithography (Figure 4D). Using this technique, 3D peptide patterns can be laser-written into the gel at cell-sized relevant length scales $(1-100 \mu \mathrm{m})$, highlighting the potential to introduce the RGD peptide or other bioactive cues spatially and temporally in a user-defined manner.

In summary, we here demonstrate the use of a cyclic disulfide, DT, as a latent thiol that participates in highly efficient and byproduct-free light-mediated reaction with NB in the presence of a photoinitiator to form cytocompatible hydrogel materials. The DT moiety yields a bifurcated crosslinker through ring opening upon light activation, that can efficiently cross-link with itself and with $\mathrm{NB}$ forming both reversible and irreversible cross-links and materials with selfhealing character in one-pot. Importantly, linear polymer precursors can be used to prepare hydrogels using the reaction between DT and NB, and their mechanics or functionality can be controlled spatiotemporally using light. Hence, we envisage that the combination of these units can open the door to exploit the use of linear polymer scaffolds that are synthetically accessible and economical for manifold biomedical applications involving cells.

\section{ASSOCIATED CONTENT}

\section{SI Supporting Information}

The Supporting Information is available free of charge at https://pubs.acs.org/doi/10.1021/acsmacrolett.0c00175.

Experimental details, characterization, and additional figures and tables (PDF)

\section{AUTHOR INFORMATION}

\section{Corresponding Author}

Roxanne E. Kieltyka - Department of Supramolecular and Biomaterials Chemistry, Leiden Institute of Chemistry, 2300 RA Leiden, The Netherlands; 이이이.org/0000-0001-91521810; Email: r.e.kieltyka@chem.leidenuniv.nl

\section{Authors}

Ciqing Tong - Department of Supramolecular and Biomaterials Chemistry, Leiden Institute of Chemistry, 2300 RA Leiden, The Netherlands

Joeri A. J. Wondergem - Huygens-Kamerlingh Onnes Laboratory, Leiden Institute of Physics, Leiden University, 2300 RA Leiden, The Netherlands; (1) orcid.org/0000-0003-00214220

Doris Heinrich - Huygens-Kamerlingh Onnes Laboratory, Leiden Institute of Physics, Leiden University, 2300 RA Leiden, The Netherlands; Fraunhofer Institute for Silicate Research ISC, 97082 Würzburg, Germany

Complete contact information is available at: https://pubs.acs.org/10.1021/acsmacrolett.0c00175

\section{Author Contributions}

All authors have given approval to the final version of the manuscript.

\section{Notes}

The authors declare no competing financial interest.

\section{ACKNOWLEDGMENTS}

We thank G. E. M. Lamers for assisting with SEM imaging, M. J. Shen for help with peptide synthesis, and A. Kros for essential discussions. C. Q. Tong thanks the China Scholarship Council for a CSC scholarship. R. E. Kieltyka thanks The Netherlands Organization for Scientific Research (NWO) for financial support (NWO-ECHO-STIP Grant 717.014.005, NWO-VENI Grant 722.012.011).

\section{REFERENCES}

(1) Huang, G.; Li, F.; Zhao, X.; Ma, Y.; Li, Y.; Lin, M.; Jin, G.; Lu, T. J.; Genin, G. M.; Xu, F. Chem. Rev. 2017, 117 (20), 12764-12850.

(2) Zhu, J. Biomaterials 2010, 31 (17), 4639-4656.

(3) Lin, C. C. RSC Adv. 2015, 5 (50), 39844-398583.

(4) Liu, S. Q.; Tay, R.; Khan, M.; Rachel Ee, P. L.; Hedrick, J. L.; Yang, Y. Y. Soft Matter 2010, 6 (1), 67-81.

(5) He, L.; Szopinski, D.; Wu, Y.; Luinstra, G. A.; Theato, P. ACS Macro Lett. 2015, 4 (7), 673-678.

(6) Casuso, P.; Perez-San Vicente, A.; Iribar, H.; Gutierrez-Rivera, A.; Izeta, A.; Loinaz, I.; Cabanero, G.; Grande, H. J.; Odriozola, I.; Dupin, D. Chem. Commun. 2014, 50 (96), 15199-15201.

(7) Wang, D.; Yang, X.; Liu, Q.; Yu, L.; Ding, J. J. Mater. Chem. B 2018, 6 (38), 6067-6079.

(8) Ren, K.; Li, B.; Xu, Q.; Xiao, C.; He, C.; Li, G.; Chen, X. Polym. Chem. 2017, 8 (45), 7017-7024.

(9) Moriyama, K.; Wakabayashi, R.; Goto, M.; Kamiya, N. Biochem. Eng. J. 2015, 93, 25-30.

(10) Nguyen, Q. T.; Hwang, Y.; Chen, A. C.; Varghese, S.; Sah, R. L. Biomaterials 2012, 33 (28), 6682-6690.

(11) Cruise, G. M.; Scharp, D. S.; Hubbell, J. A. Biomaterials 1998, 19, 1287-1294.

(12) Kharkar, P. M.; Kiick, K. L.; Kloxin, A. M. Chem. Soc. Rev. 2013, 42 (17), 7335-7372.

(13) Seliktar, D. Science 2012, 336, 1124-1128.

(14) Azagarsamy, M. A.; Anseth, K. S. ACS Macro Lett. 2013, 2 (1), $5-9$.

(15) Stenzel, M. H. ACS Macro Lett. 2013, 2 (1), 14-18.

(16) Hoyle, C. E.; Lowe, A. B.; Bowman, C. N. Chem. Soc. Rev. 2010, 39 (4), 1355-1387.

(17) Boyd, D. A. Angew. Chem., Int. Ed. 2016, 55 (50), 1548615502.

(18) Hoyle, C. E.; Bowman, C. N. Angew. Chem., Int. Ed. 2010, 49 (9), 1540-1573.

(19) Mutlu, H.; Ceper, E. B.; Li, X.; Yang, J.; Dong, W.; Ozmen, M. M.; Theato, P. Macromol. Rapid Commun. 2019, 40 (1), 1800650.

(20) Su, J. Gels 2018, 4 (3), 72.

(21) Perera, M. M.; Ayres, N. Polym. Chem. 2017, 8 (44), 67416749.

(22) Nimmo, C. M.; Shoichet, M. S. Bioconjugate Chem. 2011, 22 (11), 2199-2209.

(23) Fairbanks, B. D.; Schwartz, M. P.; Halevi, A. E.; Nuttelman, C. R.; Bowman, C. N.; Anseth, K. S. Adv. Mater. 2009, 21 (48), 50055010.

(24) Goethals, F.; Frank, D.; Du Prez, F. Prog. Polym. Sci. 2017, 64, $76-113$.

(25) Fang, J.; Ye, S. H.; Wang, J.; Zhao, T.; Mo, X.; Wagner, W. R. Biomacromolecules 2015, 16 (5), 1622-1633.

(26) Tang, H.; Tsarevsky, N. V. Polym. Chem. 2015, 6 (39), 69366945.

(27) Sakai, N.; Matile, S. J. Am. Chem. Soc. 2011, 133 (46), 1854218545.

(28) Morelli, P.; Matile, S. Helv. Chim. Acta 2017, 100 (3), No. e1600370.

(29) Hu, J.; Wu, T.; Zhang, G.; Liu, S. J. Am. Chem. Soc. 2012, 134 (18), 7624-7627.

(30) Margulis, K.; Zhang, X.; Joubert, L. M.; Bruening, K.; Tassone, C. J.; Zare, R. N.; Waymouth, R. M. Angew. Chem., Int. Ed. 2017, 56 (51), 16357-16362.

(31) Yu, H.; Wang, Y.; Yang, H.; Peng, K.; Zhang, X. J. Mater. Chem. B 2017, 5 (22), 4121-4127. 
(32) Barcan, G. A.; Zhang, X.; Waymouth, R. M. J. Am. Chem. Soc. 2015, 137 (17), 5650-5653.

(33) Song, L.; Zhang, B.; Gao, G.; Xiao, C.; Li, G. Eur. Polym. J. 2019, 115, 346-355.

(34) Tran, Y. H.; Rasmuson, M. J.; Emrick, T.; Klier, J.; Peyton, S. R. Soft Matter 2017, 13 (47), 9007-9014.

(35) Mishra, D.; Wang, S.; Michel, S.; Palui, G.; Zhan, N.; Perng, W.; Jin, Z.; Mattoussi, H. Phys. Chem. Chem. Phys. 2018, 20 (6), 3895-3902.

(36) Kisanuki, A.; Kimpara, Y.; Oikado, Y.; Kado, N.; Matsumoto, M.; Endo, K. J. Polym. Sci., Part A: Polym. Chem. 2010, 48 (22), 5247-5253.

(37) Fairbanks, B. D.; Singh, S. P.; Bowman, C. N.; Anseth, K. S. Macromolecules 2011, 44 (8), 2444-2450. 